\title{
Perfis de Idosos Praticantes de Atividades Prazerosas: características sociodemográficas, vulnerabilidade social e funcionalidade
}

\author{
Heloisa Gonçalves Ferreira ${ }^{1}$ \\ Elizabeth Joan Barbam² \\ Felipe Costa Araújo ${ }^{3}$ \\ ${ }^{1}$ Universidade Federal do Triângulo Mineiro, Uberaba, MG \\ ${ }^{2}$ Universidade Federal de São Carlos, São Carlos, São Paulo, Brasil \\ ${ }^{3}$ Universidade de São Paulo, São Paulo, SP
}

\begin{abstract}
Resumo
Este estudo mapeou perfis de idosos praticantes de atividades prazerosas e relacionou tais perfis com variáveis sociodemográficas e estado funcional. Idosos $(n=330)$ sem comprometimento cognitivo, residentes da comunidade e com diferentes níveis de vulnerabilidade social (acessado pelo Índice Paulista de Vulnerabilidade Social) responderam um questionário sociodemográfico, a escala de Pfeffer (funcionalidade) e o OPPES-BR (prática de atividades prazerosas). Por meio da análise de cluster combinando-se o método hierárquico e não-hierárquico foram identificados cinco perfis de idosos: muito ativos; muito ativos sociáveis; minimalistas; pouco ativos contemplativos; e pouco ativos intelectuais. Manter alta frequência em diversos tipos de atividades prazerosas associou-se à maior escolaridade, menor vulnerabilidade social e menor dependência funcional. O perfil dos pouco ativos contemplativos foi o mais vulnerável, com maior declínio funcional, vulnerabilidade social e taxa de analfabetismo. Diante da diversidade de perfis de idosos, são necessárias políticas públicas favorecedoras ao acesso a atividades prazerosas, principalmente aos mais vulneráveis.
\end{abstract}

Palavras-chave: idosos; estado funcional; atividades de lazer

Profiles of Older People Who Practice Pleasant Events: sociodemographic aspects, social vulnerability and functionality

\begin{abstract}
This study mapped profiles of older adults who participated in pleasant events, and related these profiles with sociodemographic and functional state. Older adults $(n=330)$ without cognitive impairments, community residents and with different social vulnerability levels (accessed by the Paulista Index of Social Vulnerability) answered a sociodemographic questionnaire, the Pfeffer Scale (functionality) and the OPPES-BR (involvement in pleasant activities). By applying a cluster analysis, which combined the hierarchical and non-hierarchical method, five profiles of older adults were identified: highly active; highly socially active; minimalists; little active contemplative; and little active intellectual. Maintaining high frequency in pleasant activities was associated with a higher education, lower social vulnerability, and less functional dependence. The little active contemplative was the most vulnerable profile, with higher scores on functional decline, social vulnerability, and illiteracy rates. Considering the diversity of the older adult's profiles, public policies favoring the access to pleasant events are needed, especially for the most vulnerable ones.

Keywords: older people; functional state; leisure activities
\end{abstract}

Perfiles de Ancianos Practicantes de Actividades Placenteras: características sociodemográficas, vulnerabilidad social y funcionalidad

\section{Resumen}

Este estudio diseñó perfiles de ancianos que practican actividades placenteras y relacionó estos perfiles con variables sociodemográficas y estado funcional. Los ancianos $(\mathrm{n}=330)$ sin comprometimiento cognitivo, residentes de la comunidad y con diferentes niveles de vulnerabilidad social (accesado por el Índice Paulista de Vulnerabilidad Social) respondieron un cuestionario sociodemográfico, Escala de Pfeffer (funcionalidad) y el OPPES-BR (práctica de actividades placenteras). Por medio de análisis de Cluster, combinando el método jerárquico y no-jerárquico, fueron identificados cinco perfiles de ancianos: muy activos; muy activos sociables; minimalistas; poco activos contemplativos; y poco activos intelectuales. Mantener alta frecuencia en diversas actividades placenteras presentó una asociación con mayor escolaridad, menor vulnerabilidad social y menor dependencia funcional. El perfil de los poco activos contemplativos fue el más vulnerable, con mayor declive funcional, vulnerabilidad social y tasa de analfabetismo. Ante la diversidad de perfiles de ancianos, son necesarias políticas públicas que favorezcan el acceso a actividades placenteras, principalmente a los más vulnerables.

Palabras clave: ancianos; estado funcional; actividades placenteras 


\section{Introdução}

No Brasil atualmente vivenciamos uma realidade de grande crescimento da população com 60 anos ou mais. A estimativa é de que esta população represente cerca de 30\% da população total brasileira em 2050 (IBGE, 2013). O crescimento da população idosa no contexto brasileiro traz diversas repercussões de cunho social, político, econômico e cultural, que sinalizam ainda muitos desafios a serem superados (Moura \& Souza, 2012; Silva, Silveira, \& Freitas, 2017). Um deles, refere-se a quais seriam as formas mais adequadas e viáveis de garantir melhores condições de saúde física e mental, com níveis maiores de qualidade de vida e bem- estar a esta população.

Recentemente o construto prática de atividades prazerosas (PAP) em idosos tem sido estudado no Brasil (Ferreira \& Barham, 2011; Ferreira, Barham, \& Fontaine, 2015; Ferreira \& Barham, 2018). Trata-se de um construto que começou a ser investigado nos Estados Unidos na década de 70 no contexto do desenvolvimento de psicoterapias comportamentais e cognitivo-comportamentais para tratar a depressão (Lewinsohn \& Libet, 1972; Lewinsohn \& Graf, 1973; Lewinsohn \& MacPhillamy, 1974). Atualmente, o envolvimento em atividades prazerosas tem sido definido como a prática de atividades ou vivências de eventos que tragam sentimentos positivos ao idoso, tendo por base dois parâmetros: frequência em atividades potencialmente agradáveis e prazer experimentado ao envolver-se na atividade (Rider, Gallagher-Thompson, \& Thompson, 2016).

A definição de PAP pode apresentar certa semelhança ao conceito de atividades de lazer, uma vez que as atividades de lazer são buscadas tendo em vista o prazer que podem possibilitar ao indivíduo que as realiza. Desta forma, o prazer experimentado pelo indivíduo na prática de uma atividade de lazer é um dos parâmetros, de caráter subjetivo, considerado na definição do conceito de lazer (Melo \& Alves, 2012). No entanto, o conceito de PAP e atividades de lazer diferem pelo fato de que no contexto de lazer, as atividades são livres de obrigação e não perpassam as esferas das atividades laborais, religiosas, domésticas e fisiológicas (Melo \& Alves, 2012). No caso da PAP, qualquer atividade que pressuponha a experiência subjetiva do prazer, seja atividade de lazer, doméstica ou laboral, é considerada uma atividade prazerosa (Ferreira \& Barham, 2011).

Muitas são as razões que fomentaram a investigação da PAP na população idosa, pois existem diversas evidências na literatura de que a PAP está associada a menores chances de desenvolvimento de depressão (Rider et al., 2016; Ferreira \& Barham, 2018), associase à expressão de sentimentos positivos (Meeks, Young, \& Looney, 2007) e a melhores níveis de funcionalidade (Pinto \& Neri, 2013; Ferreira \& Barham, 2018). Logo, entender de que forma este construto se manifesta na população idosa traz repercussões práticas para o desenvolvimento de intervenções que visem melhorar o bem-estar e a qualidade de vida desta população.

Um instrumento válido e adaptado ao contexto brasileiro para a investigação da PAP em idosos já foi disponibilizado (Ferreira \& Barham, 2013; Ferreira et al., 2015, Ferreira \& Barham, 2017). Trata-se do OPPES-BR, um instrumento que avalia frequência em atividades potencialmente agradáveis e o nível de prazer experimentado em cada atividade, além de discriminar domínios ou tipos específicos de atividades prazerosas em que o idoso pode se envolver (Ferreira \& Barham, 2017). Este instrumento foi desenvolvido inicialmente em contexto clínico para tratamento de depressão, por auxiliar na tarefa de identificar em quais os domínios de atividades prazerosas o cliente apresenta baixa frequência, e desta forma ser uma ferramenta útil para traçar um plano de intervenção (Rider et al., 2016). No entanto, o instrumento também apresenta grande utilidade para ser aplicado em contextos de pesquisas que visam investigar questões relacionadas à saúde e ao bem -estar de idosos (Ferreira \& Barham, 2018).

Identificou-se que quando aplicado em amostra de idosos brasileiros, o OPPES-BR discriminou quatro tipos ou domínios de atividades potencialmente agradáveis: Atividades Sociais e de Competência; Atividades Contemplativas; Atividades Práticas e Atividades Intelectuais (Ferreira \& Barham, 2017). Atividades Sociais e de Competência consistem em atividades que descrevem interações do idoso com seu ambiente e com outras pessoas. Tais atividades apresentam a função de socializar, mas também exprimem um senso de competência, utilidade, autoconfiança e autonomia do idoso no que concerne ao relacionamento com seu meio (viajar, conhecer novos lugares, visitar museu, aprender novos assuntos, etc) e também com as outras pessoas (ser elogiado, fazer uma nova amizade, dizer algo de forma clara, ser requisitado a ajudar e dar conselhos, etc) (Ferreira \& Barham, 2017).

As atividades contemplativas descrevem atividades de contato com a natureza (ouvir pássaros cantando, olhar para o céu, etc) e também de expressão de sentimentos positivos (pensar em pessoas que gosto, ter 
momentos de sossego, etc), porém numa perspectiva mais contemplativa, introvertida e de recolhimento. São atividades que o idoso geralmente realiza sozinho (Ferreira \& Barham, 2017).

O domínio das atividades práticas inclui atividades de envolvimento com a comunidade (ex: fazer trabalho voluntário ou comunitário, ir à igreja), mas também outras atividades que podem fazer parte da rotina doméstica (cozinhar, colecionar receitas, planejar a rotina). Esse domínio descreve eventos em que o idoso parece manter-se ativo e envolvido em atividades práticas relevantes para si e que também repercutem em sua comunidade. Por fim, as atividades intelectuais consistem apenas em atividades que pressupõem habilidades de leitura e escrita, tais como como fazer palavras cruzadas, ler livros e revistas (Ferreira \& Barham, 2017).

Tipos específicos de atividades prazerosas podem apresentar diversas funções para o idoso, uma vez que são observadas relações distintas entre tipos de atividades com outras variáveis de interesse, tais como variáveis sociodemográficas e de saúde. Por exemplo, um estudo holandês (Toepoel, 2013) com 5.910 respondentes identificou que atividades tais como trabalho voluntário, atividades culturais, fazer esportes, ler livros e fazer compras são atividades preditoras de conexão social para idosos, ao passo que atividades mais passivas (ver TV, escutar rádio e ficar no computador) não apresentaram nenhuma relação com conexão social. Também foi observado que indivíduos com 75 anos ou mais praticaram significativamente menos atividades de lazer quando comparados aos grupos etários mais jovens.

Outro estudo realizado no Canadá também encontrou diferenças significativas da prática de atividades quando se leva em consideração a idade do idoso (Gilmour, 2012). Nesta investigação, verificou-se também que idosos mais velhos praticaram significativamente menos atividades do que idosos mais jovens. Além disso, este estudo demonstrou que o envolvimento em certos tipos de atividades pode variar de acordo com o sexo do idoso. Nesse sentido, observou-se que mulheres se envolvem significativamente mais em atividades educacionais, religiosas e que requerem contato com familiares e amigos, ao passo que os homens praticam mais atividades de esportes do que as mulheres.

Já um estudo brasileiro observou que numa amostra de 337 idosos não institucionalizados e sem comprometimento cognitivo, atividades que envolvem contato social apresentam relação com depressão e com dependência nas atividades instrumentais de vida diária (AIVD’s), no sentido de que idosos que se envolviam mais em atividades que pressupõem contato com outras pessoas apresentaram menor risco para desenvolvimento de depressão e de dependência nas AIVD's, ao passo que atividades que não pressupõem contato social (por exemplo, atividades de leitura e escrita) não apresentaram relação direta com depressão e funcionalidade (Ferreira \& Barham, 2018). Logo, observa-se que a prática de diferentes tipos de atividades pode estar associada a diferentes características sociodemográficas e de saúde entre idosos, refletindo muito possivelmente, a heterogeneidade das trajetórias do envelhecimento.

Nesse sentido, estudos que investiguem a PAP em idosos brasileiros são muito promissores à medida em que permitem mapear o envolvimento dos idosos em diferentes tipos de atividades e identificar quais são as relações existentes entre PAP e características sociodemográficos e de saúde. Estudos desta natureza são ainda úteis para a identificação de condições sociodemográficas e de saúde que sejam desfavoráveis à PAP, ajudando a identificar perfis de risco. Logo, o objetivo principal deste estudo é mapear os perfis de idosos praticantes de atividades prazerosas e relacionar tais perfis com variáveis sociodemográficas e estado funcional.

\section{Método}

\section{Participantes}

Este estudo quantitativo e exploratório de corte transversal, foi realizado com 330 idosos não-institucionalizados, provenientes de uma cidade de médio porte do Estado de São Paulo. Os idosos foram selecionados de acordo com a distribuição do Índice Paulista de Vulnerabilidade Social (IPVS) na cidade, para garantir a variabilidade socioeconômica da amostra. A amostra foi composta com uma proporção de participantes de cada nível de IPVS, segundo a distribuição do IPVS do município. O IPVS é usado para classificar os setores censitários do Estado de São Paulo, de acordo com sua vulnerabilidade social. A vulnerabilidade social é um construto multidimensional que diz respeito ao processo de estar em risco para redução da qualidade de vida, em função do contexto de moradia, resultante de condições inadequadas nos planos econômico, social, psicológico, familiar, cognitivo ou físico (Fundação SEADE, 2010). Segundo o IPVS, foram estabelecidos seis níveis de vulnerabilidade social: 1 (nenbuma vulnerabilidade), 2 (vulnerabilidade muito baixa), 3 (vulnerabilidade baixa), 4 (vulnerabilidade média), 5 (vulnerabilidade alta), 6 (vulnerabilidade muito alta). 
No município onde foi realizado o estudo, observou-se a seguinte distribuição percentual do IPVS: nenhuma vulnerabilidade $(8,7 \%)$; vulnerabilidade muito baixa (36,3\%); vulnerabilidade baixa $(24,8 \%)$; vulnerabilidade média (17,5\%); vulnerabilidade alta $(8,4 \%)$ e vulnerabilidade muito alta $(4,2 \%)$ (Fundação SEADE, 2010).

$\mathrm{Na}$ amostra do presente estudo, buscou-se atingir uma distribuição percentual do IPVS que fosse semelhante à observada no município: nenhuma vulnerabilidade (12,5\%); vulnerabilidade muito baixa (39,9\%); vulnerabilidade baixa $(23,4 \%)$; vulnerabilidade média $(14 \%)$; vulnerabilidade alta $(6,5 \%)$ e vulnerabilidade muito alta $(3,7 \%)$.

A idade média dos participantes foi de 69,7 anos, variando de 60 a 93 anos e com desvio padrão igual a 7,31. Em relação ao sexo, $68,2 \%$ da amostra era do sexo feminino, 31,8\% do sexo masculino. Em relação à escolaridade, $11,5 \%$ dos idosos declarou não saber ler ou escrever. Todos os participantes atingiram pelo menos a pontuação mínima no Mini Exame do Estado Mental (MEEM) de acordo com sua escolaridade (Brucki, Nitrini, Caramelli, Bertolucci, \& Okamoto, 2003), indicando que todos contavam com capacidade cognitiva para responder aos instrumentos.

\section{Instrumentos}

Dados sociodemográficos. Foi preparado pelos pesquisadores um breve questionário para obter informações para descrever a composição da amostra, incluindo sexo, idade, escolaridade e estado civil.

Escala de Atividades Instrumentais da Vida Diária de Pfeffer (Pfeffer, Kuroasaki, Harrah, Chance, \& Filos, 1982). Esse instrumento é composto por 10 itens que avaliam a habilidade do idoso para executar atividades instrumentais da vida diária (AIVD), tais como, preparar refeições e fazer compras. Os escores podem variar de 0 a 30, sendo que escores mais altos significam maior dependência da ajuda de outras pessoas para a realização de atividades cotidianas. Quando adaptada e aplicada em amostra de idosos brasileiros (Assis, 2014), a escala apresentou excelente consistência interna $(\alpha=0,91)$ e correlações significativas com variáveis cognitivas e comportamentais que atestam validade de critério.

Versão brasileira do The California Older Person's Pleasant Events Schedule - OPPES-BR (Ferreira \& Barham, 2013; Ferreira \& Barham, 2017). Esse instrumento apresenta 44 itens que descrevem atividades que idosos tendem a achar agradáveis, sendo composto por uma estrutura de quatro fatores referente à subescala
Frequência: (a) Fator 1 - Atividades sociais e de competência $(\alpha=0,94)$; (b) Fator 2 - Atividades contemplativas $(\alpha=0,83)$; (c) Fator 3 - Atividades práticas $(\alpha=0,72)$; e (d) Fator 4 - Atividades intelectuais $(\alpha=0,70)$. O respondente deveria indicar a frequência com a qual realizou tais atividades no último mês, de acordo com uma escala que inclua as seguintes pontuações: 0 (nunca), 1 (1 a 6 vezes) ou 2 (mais de sete vezes). O respondente também deveria classificar o prazer subjetivo que experimentou ao realizar cada atividade, ou que experimentaria caso a tivesse realizado, por meio de uma escala que pode ser respondido da seguinte forma: 0 (não foi ou não teria sido agradável), 1 (foi on teria sido razoavelmente agradável) ou 2 (foi ou teria sido bastante agradáve). $\mathrm{O}$ instrumento apresentou correlações significativas com depressão, indicando evidências de validade externa.

\section{Procedimento}

Os participantes foram recrutados em lugares frequentados por idosos (centros comunitários, Unidades de Saúde da Família, associações de aposentados), ou contatados diretamente em suas residências, com a assistência dos profissionais que trabalhavam nas instituições frequentadas pelos participantes. Além disso, levou-se em consideração a distribuição do IPVS referente aos bairros do município para localizar os participantes, de forma a buscar uma distribuição do IPVS na amostra que fosse semelhante ao do município. Foram explicados os objetivos e as condições da pesquisa, antes do participante assinar o Termo de Consentimento Livre e Esclarecido. Depois desta etapa, o idoso respondia primeiramente ao Mini Exame do Estado Mental (MEEM). Quando era constatado que a pontuação obtida pelo idoso neste instrumento indicava ausência de comprometimento cognitivo, o participante respondia ao questionário sociodemográfico, à Escala de Atividades Instrumentais da Vida Diária de Pfeffer e ao OPPES-BR. As entrevistas ocorriam nas instituições onde o idoso foi contatado ou na residência do participante. As entrevistas foram realizadas por uma psicóloga e por uma profissional treinada com formação em estatística.

\section{Análise de dados}

Para identificar os perfis de idosos praticantes de atividades prazerosas, foi realizada uma análise de cluster considerando os tipos destas atividades a partir da subescala Frequência do OPPES-BR (Ferreira \& Barham, 2017). Foi utilizada uma combinação dos métodos hierárquicos e não-hierárquicos para análise de cluster 
(Hair, Black, Anderson, \& Tatham, 1998). O método hierárquico é usado com um propósito exploratório, e começa o agrupamento considerando que há tanto clusters quanto o número de indivíduos do banco de dados. A cada passo subsequente, os clusters mais similares são combinados em um outro cluster até que todos os indivíduos sejam agrupados em um único cluster. A medida de similaridade utilizada nesse processo foi o quadrado da distância Euclidiana (Hair et al., 1998). O método de Ward foi usado como o método de ligação, por permitir uma melhor distribuição dos indivíduos entre os clusters. Aplicando-se o método hierárquico, define-se o número de clusters que irá constituir a melhor solução. Para este propósito e considerando a dimensão da amostra, foi realizado o cálculo do $R^{2}$ (quantidade da variabilidade retida para cada solução de cluster). $\mathrm{O}$ aumento significativo no $R^{2}$ para a solução de dois clusters $(36.26 \%)$, para uma solução de três $(44.18 \%)$, quatro (49.53\%) ou cinco $(53.79 \%)$, cai após a solução de cinco clusters, sendo que o aumento para a solução de seis clusters foi residual $(56.87 \%)$. Os valores dos centroides da solução de cluster do método hierárquico foram usados para ajustar e aprimorar os resultados aplicando-se o método não-hierárquico (K-means). Para este método, é necessário definir a priori o número de clusters a ser analisado. Esse procedimento cria perfis de grupo distintos pela maximização das diferenças entre os clusters e minimizando a variância em cada cluster. A solução mais parcimoniosa que apresente melhor clareza conceitual deve ser então escolhida. As análises foram realizadas utilizando-se o software IBM SPSS versão 20.

\section{Considerações Éticas}

Este projeto de pesquisa foi encaminhado para o Comitê de Ética em Pesquisa do município onde foi realizada a pesquisa, tendo sido aprovado (CAAE: 00713712.4.0000.5504).

\section{Resultados}

\section{Perfis de idosos praticantes de atividades prazerosas}

De acordo com a análise utilizando-se o método hierárquico, soluções de três a cinco clusters poderiam ser consideradas. Logo, foram realizadas análises com o método K-means para a solução de três, quatro e cinco clusters. A solução de cinco clusters foi a escolhida por permitir uma melhor caracterização dos perfis de idosos praticantes de atividades, exibir um bom aumento na quantidade da variância explicada dos dados (53.79\%) e permitir uma boa distribuição dos indivíduos entre os clusters.

No geral, o modelo de cinco perfis (Figura 1) identifica dois grupos mais ativos de idosos, um grupo mediano e outros dois grupos de idosos pouco ativos. Os dois primeiros perfis são semelhantes por apresentarem as maiores frequências em todas as atividades, com exceção das atividades intelectuais, que é muito mais praticada pelo primeiro perfil do que pelo segundo. Logo, o primeiro perfil identificado foi nomeado como

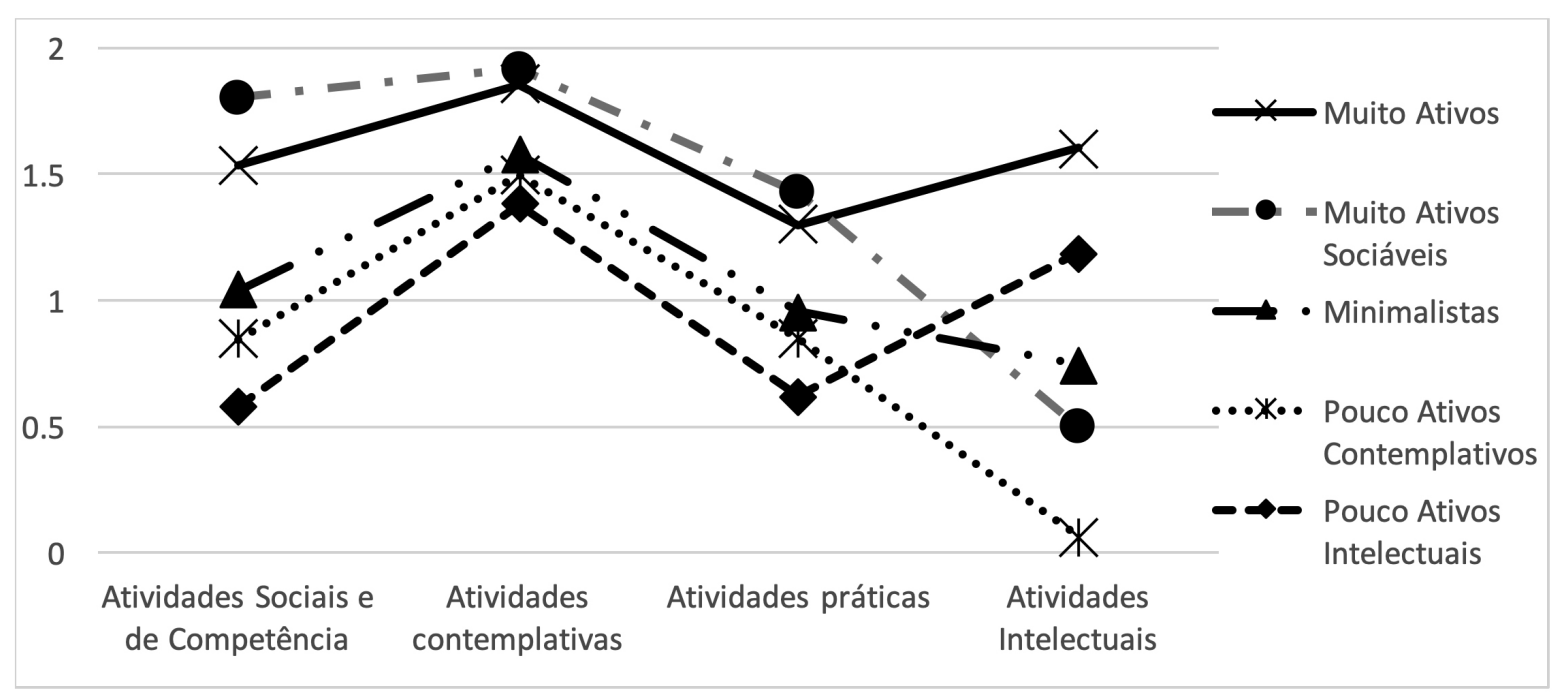

Figura 1. Representação gráfica das médias de frequência dos grupos nos quatro tipos de atividades prazerosas de acordo com os perfis de idosos praticantes. 
"Muito Ativos" ( $n=88)$, por ter frequência alta em todos os tipos de atividade, ao passo que o segundo perfil apresenta alta frequência em tipos de atividades que em sua maioria envolve contato com outras pessoas, mas frequência bastante baixa nas atividades intelectuais, e por este motivo foi nomeado como sendo o perfil de idosos "Muito Ativos Sociáveis" $(n=56)$. O terceiro perfil é caracterizado por apresentar frequência mediana em todos os tipos de atividades prazerosas, e por esta razão constitui o perfil dos idosos "Minimalistas" ( $n=71)$, que são aqueles que fazem "de tudo um pouco". Os dois últimos perfis têm em comum o baixo envolvimento na maioria das atividades, mas o que os distingue do restante dos outros grupos é que o quarto perfil apresenta ainda uma frequência moderada nas atividades contemplativas, ao passo que o quinto perfil apresenta a segunda maior média de frequência em atividades intelectuais de todos os grupos. Por estas razões, o quarto grupo foi identificado como sendo de idosos "Pouco Ativos Contemplativos" $(n=74)$, ao passo que o quinto perfil é composto por idosos "Pouco Ativos Intelectuais" $(n=41)$.

Na Tabela 1, podemos visualizar as médias de cada grupo nos tipos de atividades prazerosas. Nota-se que o Fator frequência em atividades intelectuais é a variável que melhor discrimina os clusters (apresenta o maior valor de F). De forma geral, a Tabela 1 mostra que a frequência de envolvimento em atividades sociais e de competência é significativamente diferente entre todos os perfis, sendo os Muito Ativos Sociáveis os mais envolvidos e os Pouco Ativos Intelectuais os menos envolvidos neste tipo de atividade. Nota-se que os idosos Muito Ativos e Muito Ativos Sociáveis se envolvem significativamente mais nas atividades contemplativas e nas atividades práticas, em comparação com os demais perfis. Por fim, considerando-se as frequências médias nas atividades intelectuais, observa-se que há diferenças significativas entre todos os perfis, sendo que esse tipo de atividade é mais praticado pelos Idosos Muito Ativos e também pelos Pouco Ativos Intelectuais.

\section{Relação dos perfis de idosos praticantes de atividades prazerosas com características sociodemográficas, vulnerabilidade social e estado funcional}

A Tabela 2 exibe as diferenças de média para vulnerabilidade social, idade e estado funcional entre os perfis. Observa-se que idade foi a única variável que não apresentou diferenças significativas entre os perfis. Vulnerabilidade social apresentou diferença significativa apenas para o grupo de idosos "Pouco Ativos Contemplativos" com relação aos outros perfis, indicando que este grupo foi o que apresentou maior vulnerabilidade social. Nota-se diferença significativa com relação ao estado funcional apenas para os perfis dos idosos pouco

Tabela 1.

Frequência média nos tipos de atividades prazerosas entre os perfis de idosos praticantes $(N=330)$

\begin{tabular}{|c|c|c|c|c|c|c|}
\hline Fatores & $\begin{array}{l}\text { Muito Ativos } \\
\qquad(N=88)\end{array}$ & $\begin{array}{l}\text { Muito Ativos } \\
\text { Sociáveis } \\
(N=56)\end{array}$ & $\begin{array}{c}\text { Minimalistas } \\
\quad(N=71)\end{array}$ & $\begin{array}{c}\text { Pouco Ativos } \\
\text { Contemplativos } \\
(N=74)\end{array}$ & $\begin{array}{c}\text { Pouco ativos } \\
\text { Intelectuais } \\
\quad(N=41)\end{array}$ & $F^{*}$ \\
\hline $\begin{array}{l}\text { Frequência em } \\
\text { atividades sociais } \\
\text { e de competência }\end{array}$ & $1.54_{a}$ & $1.80_{\mathrm{b}}$ & $1.04_{c}$ & $.84_{d}$ & $.58_{\mathrm{e}}$ & 149.155 \\
\hline $\begin{array}{l}\text { Frequência } \\
\text { em atividades } \\
\text { contemplativas }\end{array}$ & $1.85_{\mathrm{a}}$ & $1.92_{\mathrm{a}}$ & $1.57_{\mathrm{b}}$ & $1.49_{b, c}$ & $1.38_{c}$ & 50.735 \\
\hline $\begin{array}{l}\text { Frequência } \\
\text { em atividades } \\
\text { práticas }\end{array}$ & $1.30_{\mathrm{a}}$ & $1.43_{\mathrm{a}}$ & $.95_{\mathrm{b}}$ & $.84_{b}$ & $.62_{c}$ & 55.395 \\
\hline $\begin{array}{l}\text { Frequência } \\
\text { em atividades } \\
\text { intelectuais }\end{array}$ & $1.60_{\mathrm{a}}$ & $.50_{\mathrm{b}}$ & $.75_{c}$ & $.06_{d}$ & $1.19_{\mathrm{e}}$ & 207.049 \\
\hline
\end{tabular}

Notas. A escala de Frequência variou de 0 a 2. As médias com diferentes letras subscritas por linha diferem significativamente $(p<0.05)$.

*Para todos os valores de $F, p<0.001$ 
Tabela 2.

Relações dos perfis de idosos praticantes de atividades prazerosas com vulnerabilidade social, idade e estado funcional

\begin{tabular}{|c|c|c|c|c|c|c|}
\hline & & Muito Ativos & $\begin{array}{l}\text { Muito Ativos } \\
\text { Sociáveis }\end{array}$ & Minimalistas & $\begin{array}{c}\text { Pouco Ativos } \\
\text { Contemplativos }\end{array}$ & $\begin{array}{c}\text { Pouco ativos } \\
\text { Intelectuais }\end{array}$ \\
\hline $\begin{array}{l}\text { Vulnerabilidade Social } \\
\text { (IPVS) }\end{array}$ & $\begin{array}{c}M \\
(D P)\end{array}$ & $\begin{array}{l}2.56_{a} \\
(1.07)\end{array}$ & $\begin{array}{l}2.59{ }_{\mathrm{a}} \\
(.91)\end{array}$ & $\begin{array}{l}2.72_{\mathrm{a}} \\
(1.27)\end{array}$ & $\begin{array}{l}3.28_{\mathrm{b}} \\
(1.45)\end{array}$ & $\begin{array}{l}2.29 \\
(1.18)\end{array}$ \\
\hline Idade & $\begin{array}{c}M \\
(D P)\end{array}$ & $\begin{array}{l}68.85 \\
(6.26)\end{array}$ & $\begin{array}{l}70.13 \mathrm{a} \\
(7.22)\end{array}$ & $\begin{array}{l}70.088_{a} \\
(7.46)\end{array}$ & $\begin{array}{l}69.27_{\mathrm{a}} \\
(7.65)^{2}\end{array}$ & $\begin{array}{l}70.80 a \\
(8.34)\end{array}$ \\
\hline $\begin{array}{l}\text { Funcionalidade } \\
\text { (Índice Pfeffer) }\end{array}$ & $\begin{array}{c}M \\
(D P)\end{array}$ & $\begin{array}{l}.14_{a} \\
(.61)\end{array}$ & $\begin{array}{l}0.38 \text { a } \\
(2.01)\end{array}$ & $\begin{array}{l}1.03 \mathrm{a} \\
(2.22)\end{array}$ & $\begin{array}{l}2.23 \mathrm{~b} \\
(3.08)\end{array}$ & $\begin{array}{l}2.45_{b} \\
(4.95)\end{array}$ \\
\hline
\end{tabular}

Nota. As médias com diferentes letras subscritas por linha diferem significativamente $(p<0.05)$.

$\mathrm{M}=$ Média

DP $=$ Desvio Padrão

IPVS = Índice Paulista de Vulnerabilidade Social

ativos (pouco ativos contemplativos e intelectuais), em relação a todos os outros perfis, indicando que idosos menos envolvidos em atividades prazerosas apresentam maior dependência funcional quando comparado aos outros grupos.

A Tabela 3 apresenta a distribuição para sexo e escolaridade entre os perfis. Através de análises com o teste do Qui-Quadrado, foi constatado que os perfis diferem significativamente com relação ao sexo e à escolaridade. Uma análise mais cuidadosa da Tabela 3, revela que as mulheres apareceram muito mais representadas do que o esperado, no perfil dos Muito Ativos Sociáveis. Da mesma forma, também é possível notar que com relação à escolaridade, idosos analfabetos estão menos representados no perfil dos Muito Ativos, porém muito mais representados no perfil dos Pouco Ativos Contemplativos. Já os idosos mais escolarizados, estão muito mais representados no perfil dos Pouco Ativos Intelectuais. Observa-se ainda que no grupo dos Muito Ativos Sociáveis, os idosos com maior escolaridade são os menos representados.

\section{Discussão}

Este estudo teve por objetivo mapear perfis de idosos praticantes de atividades prazerosas e relacioná-los a condições sociodemográficas (sexo, idade, escolaridade e vulnerabilidade social) e de saúde (estado funcional). Observou-se que foram mapeados cinco tipos de perfis de idosos: (a) idosos muito ativos, que apresentaram frequência alta em todos os tipos de atividades; (b) idosos muito ativos sociáveis, que apresentaram maior frequência nas atividades sociais e de competência e também frequência alta nos outros tipos de atividade, exceto nas atividades intelectuais; (c) idosos minimalistas, que apresentaram frequência mediana em todos os tipos de atividades; (d) idosos pouco ativos contemplativos, que apresentaram baixa frequência em todos os tipos de atividade, porém frequência mediana nas atividades contemplativas; e (e) idosos pouco ativos intelectuais, que apresentaram baixa frequência em todos os tipos de atividade, mas frequência alta nas atividades intelectuais.

Os grupos dos idosos muito ativos e muito ativos sociáveis apresentaram maior frequência na maioria dos domínios de atividade. Entretanto, o grupo de idosos muito ativos sociáveis se diferenciaram dos outros grupos por duas características principais: esses idosos frequentavam atividades sociais e de competência com maior frequência dentre todos os outros grupos, porém com baixa frequência nas atividades intelectuais. Este perfil também apresentou níveis mais baixos de escolaridade, quando comparado aos outros perfis, o que poderia em parte explicar a baixa frequência nas atividades intelectuais. Entretanto, ao analisar as características deste perfil, ter pouca escolaridade e não se envolver em 
Tabela 3.

Relações dos perfis dos idosos praticantes de atividades prazerosas com sexo e escolaridade

\begin{tabular}{|c|c|c|c|c|c|c|c|c|}
\hline & & & $\begin{array}{l}\text { Muito } \\
\text { Ativos } \\
(26.7 \%)\end{array}$ & $\begin{array}{l}\text { Muito } \\
\text { Ativos } \\
\text { Sociáveis } \\
(17 \%)\end{array}$ & $\begin{array}{c}\text { Minimalistas } \\
(21.5 \%)\end{array}$ & $\begin{array}{c}\text { Pouco Ativos } \\
\text { Contemplativos } \\
(22.4 \%)\end{array}$ & $\begin{array}{c}\text { Pouco } \\
\text { ativos } \\
\text { Intelectuais } \\
(12.4 \%)\end{array}$ & $X^{2}$ \\
\hline \multirow[t]{6}{*}{ Sexo } & \multirow[t]{3}{*}{ Feminino } & $\mathrm{N}$ & 61 & 47 & 44 & 51 & 22 & \multirow[t]{6}{*}{$11.72^{*}$} \\
\hline & & $(\%)$ & 27.1 & 20.9 & 19.6 & 22.7 & 9.8 & \\
\hline & & $\mathrm{RP}^{\mathrm{a}}$ & (0.1) & (1.4) & $(-0.6)$ & (0.1) & $(-1.1)$ & \\
\hline & \multirow[t]{3}{*}{ Masculino } & $\mathrm{N}$ & 27 & 9 & 27 & 23 & 19 & \\
\hline & & $(\%)$ & 25.7 & 8.6 & 25.7 & 21.9 & 18.1 & \\
\hline & & $\mathrm{RP}^{\mathrm{a}}$ & $(-0.2)$ & $(-2.1)$ & $(0.9)$ & $(-0.1)$ & (1.6) & \\
\hline \multirow[t]{9}{*}{ Escolaridade } & \multirow[t]{3}{*}{ Analfabeto } & $\mathrm{N}$ & 2 & 11 & 3 & 20 & 3 & \multirow{6}{*}{$66.43^{*}$} \\
\hline & & $(\%)$ & 5.1 & 28.2 & 7.7 & 51.3 & 7.7 & \\
\hline & & $\mathrm{RP}^{\mathrm{a}}$ & $(-2.6)$ & $(1.7)$ & $(-1.9)$ & (3.8) & $(-0.8)$ & \\
\hline & \multirow{3}{*}{$\begin{array}{l}\text { Até } 8 \text { anos } \\
\text { de estudo }\end{array}$} & $\mathrm{N}$ & 56 & 38 & 45 & 47 & 13 & \\
\hline & & $(\%)$ & 28.1 & 19.1 & 22.6 & 23.6 & 6.5 & \\
\hline & & $\mathrm{RP}^{\mathrm{a}}$ & (.4) & $(.7)$ & (.3) & (.4) & $(-2.4)$ & \\
\hline & \multirow{3}{*}{$\begin{array}{l}\text { Mais de } \\
8 \text { anos de } \\
\text { estudo }\end{array}$} & $\mathrm{N}$ & 30 & 7 & 23 & 7 & 25 & \\
\hline & & $(\%)$ & 32.6 & 7.6 & 25.0 & 7.6 & 27.2 & \\
\hline & & $\mathrm{RP}^{\mathrm{a}}$ & (1.1) & $(-2.2)$ & $(0.7)$ & $(-3.0)$ & $(4.0)$ & \\
\hline
\end{tabular}

Notas: ${ }^{a}$ Valores nos parêntesis representam os resíduos padrão (RP). Valores $>1.96$ em valor absoluto indicam que há mais (ou menos) casos na célula, do que haveria no caso das variáveis serem independentes.

$* p<0.05$.

atividades intelectuais não pressupõe baixa frequência em outros tipos de atividades prazerosas, uma vez que este perfil apresentou alta frequência nos demais tipos de atividades.

Já o perfil de idosos Pouco Ativos Intelectuais representou o oposto do perfil dos idosos Muito Ativos Sociáveis, por apresentar maior escolaridade e maior frequência nas atividades intelectuais, porém baixa frequência nos demais tipos de atividade. Estes resultados sugerem que o envolvimento de idosos em tipos distintos de atividades pode ser influenciado por variáveis sociodemográficas, tais como escolaridade. No entanto, baixa escolaridade não necessariamente implicaria em pouco envolvimento em todos os tipos de atividades prazerosas, assim como alta escolaridade também não implicaria em alto envolvimento em todos os tipos de atividades prazerosas, como evidenciado pelas características dos perfis dos idosos Muito Ativos Sociáveis e dos Muito Ativos Intelectuais. Tais resultados contrapõem, em parte, os achados de Moura e Souza (2012), que apontaram em revisão de literatura que os estudos nacionais sobre esta temática têm demonstrado uma associação entre baixa escolaridade e pouco envolvimento em atividades de lazer. Os resultados do presente estudo sugerem que idosos com diferentes escolaridades podem ter preferências distintas, e por esta razão, se envolvem mais ou menos em algum domínio específico de atividade, refletindo numa heterogeneidade de perfis. 
Outra característica interessante de ser observada sobre o grupo dos idosos muito ativos sociáveis, é que trata-se de um grupo composto predominantemente por mulheres. Logo, este estudo revela que são mais as idosas do que os idosos, que têm se envolvido em atividades que pressupõem contato com outras pessoas (familiares e amigos), e que também expressam competência no relacionamento com o outro e com o seu ambiente. De fato, atualmente são as mulheres que predominam na população idosa, fenômeno conhecido como feminização da velhice (Almeida, Mafra, Silva \& Kanso, 2015). As mulheres mais velhas são também tradicionalmente consideradas como as responsáveis por manter os laços sociais no contexto familiar (kin keepers) quando comparadas aos homens mais velhos (Trotman \& Brody, 2002), o que naturalmente levaria essas mulheres a um maior envolvimento em atividades sociais quando comparadas aos homens. Tais dados são observados tanto em estudos internacionais (Gilmour, 2012) como no contexto brasileiro (Brunnet et al., 2013), sendo que este último estudo identificou no discurso de mulheres entre 60 e 70 anos, que atividades envolvendo práticas de interação em grupos com outras pessoas constituía uma das categorias mais frequentes praticadas por mulheres desta faixa etária. Logo, o presente estudo evidencia diferenças com relação ao sexo para idosos no engajamento em certos tipos de atividades prazerosas, no caso nas atividades sociais e de competência, sugerindo que o sexo também é outra variável a ser levada em consideração para se compreender o interesse do idoso no envolvimento em certos tipos de atividade.

Com relação à vulnerabilidade social, observou-se que o único grupo que se diferenciou dos demais foi o perfil dos Pouco Ativos Contemplativos, com maior vulnerabilidade social. A vulnerabilidade social tem relação com a possibilidade de acesso da população aos recursos sociais e econômicos do município (Fundação SEADE, 2010), sendo que idosos que fazem parte deste perfil pareceram ter acesso mais restrito aos vários tipos de atividades, provavelmente por apresentarem maior vulnerabilidade social. Moura e Souza (2012) apontam que a falta de espaços e opções apropriadas, dificuldades de acesso, precariedade do transporte público, falta de segurança, dificuldades de moradia e de saúde são exemplos de fatores que impedem que idosos se envolvam em atividades de lazer. Os resultados do presente estudo parecem confirmar este dado, sugerindo que a vulnerabilidade social é outra variável que pode afetar o envolvimento de idosos em atividades prazerosas.
No entanto, o grupo dos Pouco Ativos Intelectuais também se envolveu pouco em atividades prazerosas, assim como o grupo dos Pouco Ativos Contemplativos, mas não apresentou menor vulnerabilidade social quando comparados aos perfis dos idosos mais ativos. Nesse sentido, nota-se que um idoso pode ter pouco envolvimento em atividades no geral, sem que isso necessariamente represente índices altos de vulnerabilidade social. Este dado sugere que o envolvimento do idoso em atividades prazerosas seja influenciado por outras variáveis que vão além de condições sociodemográficas. Nesse sentido, Moura e Souza (2015) apontam que outros determinantes para o envolvimento em atividades de lazer envolvem também a vontade própria do idoso, aspectos interpessoais do ambiente e incentivo familiar, o que ressalta a importância de investigar a PAP considerando-se também outras variáveis para além das que foram consideradas no presente estudo.

Com relação ao estado funcional, observou-se que os perfis dos idosos pouco ativos (tanto os contemplativos quanto os intelectuais) foram os que apresentaram maior tendência à dependência funcional quando comparados aos demais perfis, corroborando a relação já bem documentada na literatura entre pior funcionalidade associada à baixa frequência em diversos tipos de atividades (D'Orsi, Xavier \& Ramos, 2011; Pinto \& Neri, 2013; Ferreira \& Barham, 2018). Pior estado funcional sinaliza condições que impedem o envolvimento em atividades no geral, tais como dores nas articulações e dificuldades de locomoção (Brunnet et al., 2013), obrigando o idoso a reduzir frequência ou até mesmo deixar de praticar certos tipos de atividades, evidenciando a grande influência que a saúde física pode desempenhar no perfil de atividades do idoso.

O grupo dos idosos Pouco Ativos Contemplativos pareceu ser o perfil mais vulnerável em termos sociodemográficos e de saúde, pois os idosos deste grupo apresentaram maior vulnerabilidade social, maior dependência funcional e maior porcentagem de analfabetos quando comparado a todos os outros grupos. Por outro lado, também podemos inferir que as atividades contemplativas seria o tipo de atividade mais provável de ser praticado por idosos que apresentam maior vulnerabilidade social, menor escolaridade e maior dependência funcional, uma vez que esse domínio é composto por atividades mais simples de serem realizadas e que também não pressupõem gastos financeiros, tais como estar em contato com a natureza e experimentar sentimentos positivos. Porém, a partir dos dados observados, podemos levantar a hipótese de 
que envolver-se medianamente em atividades contemplativas apenas, não seria suficiente para proteger os idosos de desfechos de saúde desfavoráveis, tais como declínio funcional.

Não foram identificadas diferenças significativas de idade entre os perfis de idosos. Este dado contrapõe resultados de outras pesquisas que apontaram diferenças de idade na prática de tipos específicos de atividade (Gilmour, 2012; Toepoel, 2013). Pode ser que essas diferenças de idade não tenham sido observadas neste estudo pelo fato da amostra ser composta em sua maioria por idosos mais jovens, cuja idade média não chegou nem aos 70 anos $(M=69,7)$. Futuramente seria importante controlar esta variável e garantir uma proporção maior de idosos mais velhos na amostra, para então testar se haverá diferenças de idade com relação à prática em tipos específicos de atividades.

Igualmente importante, seria a realização de investigações futuras focadas em identificar relações destes perfis com desfechos em saúde mental, como por exemplo, depressão, bem-estar subjetivo e solidão, visando determinar quais perfis seriam mais e menos vulneráveis a desfechos desfavoráveis em saúde mental.

Identificar os perfis de idosos praticantes de atividades prazerosas e relacioná-los a condições sociodemográficas e de saúde, permitiu observar a heterogeneidade das preferências, condições e características de idosos com relação à PAP. Tal diversidade de perfis, muito possivelmente reflete na heterogeneidade de trajetórias do envelhecimento, uma vez que esta fase da vida e vivenciada de forma distinta pelos indivíduos, sendo dependente de fatores genéticos, físicos, psicológicos e culturais (Rabelo \& Neri, 2014).

Conclui-se que variáveis sociodemográficas e estado funcional influenciam a PAP de idosos, entretanto, é muito provável que a escolha de envolvimento em tipos específicos de atividade também seja dependente de outras variáveis, tais como interesse, motivação e aspectos específicos relacionados ao ambiente do idoso. A influência dessas variáveis na PAP deve ser melhor investigada em estudos futuros.

Os resultados deste estudo também evidenciam a necessidade de políticas públicas que favoreçam o acesso a atividades prazerosas e de lazer principalmente aos idosos mais vulneráveis, com baixo envolvimento em atividades e que apresentam condições sociodemográficas e de saúde mais desfavoráveis. Políticas públicas voltadas a esta temática requerem o envolvimento de diversas esferas (econômica, política, cultural, social, psicológica, biológica), nesse sentido, conhecer o perfil dos idosos com pouco acesso a atividades de lazer e atividades que possam gerar prazer, é uma medida importante que pode ajudar na formulação e implementação de tais políticas públicas.

\section{Considerações Finais}

Este estudo identificou perfis diversos de PAP com características sociodemográficas e de funcionalidade variadas na população idosa, evidenciando a heterogeneidade das experiências de envelhecimento no que concerne à PAP. Variáveis sociodemográficas e estado funcional parecem influenciar o envolvimento do idoso em tipos distintos de atividades, no entanto, levanta-se a hipótese de que tal envolvimento também seja determinado por outras variáveis, tais como interesse e motivação pessoal do idoso.

Manter uma alta frequência em diversos tipos de atividades prazerosas associou-se a melhores condições sociodemográficas (maior escolaridade e menor vulnerabilidade social) e de saúde (menor dependência funcional). O perfil mais vulnerável de idosos praticantes de atividades prazerosas foi o grupo dos idosos pouco ativos contemplativos, que apresentou maior declínio funcional, maior vulnerabilidade social e maiores taxas de analfabetismo, quando comparados aos outros perfis. Logo, este estudo gera subsídios importantes para se buscar formas adequadas de planejamento e implementação de intervenções e políticas públicas voltadas à maior inserção social do idoso, que implique em possibilitar acesso a atividades que gerem prazer e bem-estar, sobretudo com a função de auxiliar os idosos que constituem o perfil de risco.

Este estudo conta com algumas limitações, como por exemplo, os dados foram coletados em uma região específica do país, sendo que a generalização dos resultados para todo o contexto brasileiro deve ser feita com cautela. Além disso, o delineamento do presente estudo não permite estabelecer relações de causa e efeito entre variáveis sociodemográficas e de saúde com a PAP em idosos. Estudos futuros devem buscar estabelecer delineamentos adequados (por exemplo, aplicações de modelos de regressão ou de equações estruturais) para buscar investigar em maior profundidade a natureza das relações entre essas variáveis. Outras variáveis também devem ser consideradas na investigação do perfil de PAP por idosos futuramente, tais como a influência de variáveis pessoais (motivação e interesse) na prática de diferentes tipos de atividades prazerosas, e também suas relações com desfechos em saúde mental.

Psico-USF, Bragança Paulista, v. 24, n. 3, p. 541-553, jul./set. 2019 


\section{Referências}

Almeida, A.V., Mafra, S.C.T., Silva, E.P., \& Kanso, S. (2015). A Feminização da Velhice: em foco as características socioeconômicas, pessoais e familiares das idosas e o risco social. Textos \& Contextos, 14(1), 115-131. doi: 10.15448/1677-9509.2015.1.19830.

Assis, L. O. (2014). Propriedades psicométricas do questionário de atividades de vida diária de Pfeffer. Tese de Doutorado, Universidade Federal de Minas Gerais/ UFMG, Belo Horizonte, MG Brasil. Recuperado de http://www.bibliotecadigital.ufmg.br/dspace/ handle/1843/BUBD-9VNG4Q.

Brucki, S. M. D., Nitrini, R., Caramelli, P., Bertolucci, P. H. F., \& Okamoto, I., H. (2003). Suggestions for utilization of the mini-mental state examination in Brazil. Arquivos de neuro-psiquiatria, 61(3B), 777-781. doi: 10.1590/S0004-282X2003000500014.

Brunnet, A. E., Andrades, B., Souza, C. S., Weber, J. L. A., Martinato, L., Loreto, T., \& Pizzinato, A. (2013). Práticas Sociais e Significados do Envelhecimento para Mulheres Idosas. Pensando Famílias, 17(1), 99-109. Recuperado de http:// pepsic.bvsalud.org/scielo.php?script=sci_arttext\&pid=S1679-494X2013000100010.

D’Orsi, E., Xavier, A. J., \& Ramos, L. R. (2011). Trabalho, suporte social e lazer protegem idosos da perda funcional: Estudo Epidoso. Revista de Saúde Pública, 45(4), 685-692. doi: 10.1590/ S0034-89102011000400007.

Ferreira, H. G., \& Barham, E. J. (2011). O envolvimento de idosos em atividades prazerosas: revisão de literatura sobre instrumentos de aferição. Revista Brasileira de Geriatria e Gerontologia, 14, 579-90. doi: 10.1590/S1809-98232011000300017.

Ferreira, H. G., \& Barham, E. J. (2013). Adaptação transcultural de um instrumento para avaliar o envolvimento de idosos em atividades prazerosas. Cadernos de Saúde Pública, 29(12), 2554-2560. doi: 10.1590/0102-311X00130212.

Ferreira, H. G., Barham E. J., \& Fontaine, A. M. G. V. (2015). A measure to assess Brazilian older people's involvement in pleasant activities: Initial evidence of internal and external validity. Clinical Gerontology, 38(5), 375-94. doi: 10.1080/07317115.2015.1067270.
Ferreira, H. G., \& Barham, E. J. (2017). Estrutura fatorial da versão brasileira do California Older Person's Pleasant Events Schedule. Avaliação Psicológica, 16(4), 405-414. doi: 10.15689/ap.2017.1604.12627.

Ferreira, H.G., \& Barham, E.J. (2018). Relationships between pleasant events, depression, functionality and socio-demographic variables in the elderly. Paidéia (Ribeirão Preto), 28, e2815. doi: 10.1590/1982-4327e2815.

Fundação SEADE. (2010). Índice Paulista de Vulnerabilidade Social. Recuperado de http:// indices-ilp.al.sp.gov.br/view/pdf/ipvs/ principais_resultados.pdf.

Gilmour, H. (2012). Social participation and the health and well-being of Canadian seniors. Health Reports, 23(4), 3-12. Recuperado de http://europepmc. org/abstract/med/23356042.

Hair, J. F., Black, W., Anderson, R.E., \& Tatham, R. L. (1998). Multivariate Data Analysis (5ed.). New Jersey: Prentice-Hall.

IBGE. (2013). Instituto Brasileiro de Geografia e Estatística. Sintese de indicadores sociais: uma análise das condições de vida da população brasileira. Recuperado de http://biblioteca.ibge.gov.br/visualizacao/livros/ liv66777.pdf.

Lewinsohn, P. M., \& Graf, M. (1973). Pleasant activities and depression. Journal of Consulting and Clinical Psychology, 41(2), 261-268. doi: 10.1037/h0035142.

Lewinsohn, P. M., \& Libet, J. (1972). Pleasant events, activity schedules and depressions. Journal of $A b$ normal Psychology, 79(3), 291-295. doi: 10.1037/ h0033207.

Lewinsohn, P. M., \& MacPhillamy, D. J. (1974). The relationship between age and engagement in pleasant Activities. Journal of Gerontology, 29(3), 290-294. doi: 10.1093/geronj/29.3.290.

Meeks, S., Young, C. M., \& Looney, S. W. (2007). Activity participation and affect among nursing home residents: Support for a behavioural model of depression. Aging \& Mental Health, 11(6), 751-760. doi: 10.1080/13607860701546910.

Melo, V.A., \& Alves, E.D., Jr. (2012). Introdução ao laz̧er (2a ed). São Paulo: Manole.

Moura, G.A., \& Souza, L.K. (2012). Autoimagem, socialização, tempo livre e lazer: quarto 
desafios à velhice. Textos \& Contextos, 11(1), 172183. Recuperado de http://www.redalyc.org/ $\mathrm{html} / 3215 / 321527331016 /$.

Moura, G.A., \& Souza, L.K. (2015). Atividades e motivação para a adesão em grupos de convivência para idosos. Estudos Interdisciplinares sobre o Envelhecimento, 20(3), 755-769. Recuperado de http:// www.seer.ufrgs.br/RevEnvelhecer/article/ view/47740/36817.

Pfeffer, R. I., Kurosaki, T. T., Harrah, C. H. J., Chance, J. M., \& Filos, S. (1982). Measurement of functional activities in older adults in the community. Journal of Gerontology, 37(7), 323-329. doi: 10.1093/ geronj/37.3.323.

Pinto, J. M., \& Neri, A. L. (2013). Doenças crônicas, capacidade funcional, envolvimento social e satisfação em idosos comunitários: Estudo Fibra. Ciência \& Saúde Coletiva, 18(12), 3449-3460. doi: 10.1590/S1413-81232013001200002.

Rabelo, D. F., \& Neri, A. L. (2014). A complexidade emocional dos relacionamentos intergeracionais e a saúde mental dos idosos. Pensando famílias, 18(1),
138-153. Recuperado de http://pepsic.bvsalud. org/pdf/penf/v18n1/v18n1a12.pdf

Rider, K., Gallagher-Thompson, D., \& Thompson, L. (2016). California Older Person's Pleasant Events Schedule: A tool to help older adults increase positive experiences. Clinical Gerontology, 39(1), 64-83. doi: 10.1080/07317115.2015.1101635.

Silva, H.S., Silveira, M.H., \& Freitas, T.H.S. (2017). Práticas gerontológicas e o desafio da Gerontologia como ciência e profissão. In. C.M.R.G. de Carvalho, \& L.F. de Araújo (Orgs.). Envelhecimento e Práticas Gerontológicas (pp.131-148). Curitiba: CRV.

Toepoel, V. (2013). Ageing, Leisure, and Social Connectedness: How could Leisure Help Reduce Social Isolation of Older People? Social Indicators Research, 113(1), 355-372. doi: 10.1007/s11205-012-0097-6.

Trotman, F.K., \& Brody, C.M. (2002). Psychotherapy and counseling with older women: Cross-cultural, family, and end-of-life issues. New York: Springer.

Recebido em: 29/05/2018

Reformulado em: 01/10/2018

Aprovado em: 05/10/2018 
Sobre os autores:

Heloísa Gonçalves Ferreira. Professora adjunta do Departamento e da Pós-Graduação em Psicologia da Universidade Federal do Triângulo Mineiro (UFTM), atuando principalmente nas áreas de: saúde mental do idoso, desenvolvimento de instrumentos psicométricos e psicoterapia comportamental. Psicóloga, Mestre e Doutora pela Universidade Federal de São Carlos (UFSCar). Especialista em terapia comportamental pelo Instituto de Terapia por Contingências de Reforçamento - ITCR de Campinas-SP.

ORCID: https://orcid.org/0000-0002-3545-937

E-mail: helogf@gmail.com

Elizabeth Joan Barham. Psicóloga, Professora associada no Departamento de Psicologia da UFSCar. Investiga o bem-estar e desenvolvimento socioemocional de adultos, especialmente em relação a sua capacidade de lidar com envolvimentos e responsabilidades familiares.

ORCID: https://orcid.org/0000-0002-7270-4918

E-mail:lisa@ufscar.br

Felipe Costa Araújo. Mestre em Administração de Organizações pela FEA-USP - Ribeirão Preto, Engenheiro de Produção graduado pela Universidade Federal de Itajubá e técnico em Administração de Empresas pela Escola Técnica de Formação Gerencial. Atua com métodos quantitativos, pesquisa operacional e métodos de tomada de decisão. ORCID: https://orcid.org/0000-0003-1284-8968

E-mail: fcaraujo@usp.br

Contato com os autores:

Rua Vigário Carlos, 100, sala 525 - Nossa Senhora da Abadia Uberaba-MG, Brasil

CEP: 38025-350 